\title{
Spatial-domain cavity ringdown from a high-finesse plane Fabry-Perot cavity
}

\author{
Jae Yong Lee, Jae Wan Kim, Yong Shim Yoo, and Jae Won Hahna) \\ Division of Optical Metrology, Korea Research Institute of Standards and Science, \\ P.O. Box 102, Yusong, Taejon 305-600, Korea \\ Hai-Woong Lee \\ Department of Physics, Korea Advanced Institute of Science and Technology, \\ 373-1 Kusong-dong Yusong-gu, Taejon 305-701, Korea
}

(Received 1 June 2001; accepted for publication 15 October 2001)

\begin{abstract}
We investigate the optical transmission of a tilted plane Fabry-Perot cavity leading to spatial cavity ringdown, the exponentially decaying intensity output present along the transverse spatial coordinate. Primary features of the spatial cavity ringdown are theoretically predicted from the spectral and spatial cavity transfer function which is derived analytically on the combined basis of ray optics and diffraction theory applied to an ideal diffraction lossless cavity of one transverse dimension. Spatial frequency filtration by a narrow Lorentzian-shaped cavity resonance is shown to play key roles on the spatial aspects of transmitted beam profiles. Our theoretical formulation is further extended to the case of wedged plane Fabry-Perot cavities. The experimental observation of spatial cavity ringdown signals exhibits an excellent agreement with the theoretical prediction.

(C) 2002 American Institute of Physics. [DOI: 10.1063/1.1425443]
\end{abstract}

\section{INTRODUCTION}

Cavity ringdown (CRD) refers to the transient of cavity photon decay that occurs subsequent to the interruption of optical excitation of a cavity. The time rate of such decay, being proportional to the total cavity loss, provides a sensitive measure of quantifying the amount of loss attributed to the cavity itself and to the sample when introduced in the cavity. The principle of cavity ringdown spectroscopy (CRDS) takes advantages of this unique detection scheme measuring the ringdown time, i.e., the decay constant of a cavity ringdown signal that decreases exponentially in time. ${ }^{1}$

During the last decade there have been widespread CRD applications, and a number of novel variations have emerged for advanced implementations. ${ }^{2-11}$ Among them is the realization of the CRD technique in "spatial" domain rather than in "time" domain, ${ }^{12}$ which features a new optical arrangement using a plane Fabry-Perot (PFP) cavity in combination with a narrow, collimated $\mathrm{cw}$ laser beam in oblique incidence as Fig. 1 illustrates. The heart of spatial cavity ringdown (SCRD) signal generation is, in a crude sense, very much analogous with the principle of the CRD technique that works with a geometrically stable cavity to have transmitted wave components undergo a constant time delay and an intensity decrease at each cavity round trip. A PFP etalon in the SCRD setup functions similarly, but produces the lateral displacement and the intensity drop of the consecutively outgoing multiple beams along the spatial coordinate instead. The decay constant of a SCRD signal is found to associate directly with the total PFP etalon loss containing a sample inside, which implies a potential of the SCRD concept for chemical sensing applications. Moreover, the SCRD adds ad-

${ }^{a)}$ Electronic mail: jwhahn@kriss.re.kr vantageous features from the spectroscopic standpoint as well as high sensitivity inherited from the conventional CRD technique; the fact that the SCRD signal is persistent in time along a spatial coordinate allows particular merits. Broadband implementation is readily compatible by incorporating a dispersive element with a two-dimensional array detector in series to obtain frequency-dependent SCRD signals in a single-shot fashion. This broadband capability would be particularly promising for in situ spectral measurements involved with industrial process control and environmental monitoring. In addition, the SCRD technique can be realized without the need for fast and sophisticated control mechanisms such as a cavity-lock servo, a cavity dither circuitry, a fast laser turn-off driver, and a rapid cavity excursion procedure that are indispensable for the conventional CRD technique using $\mathrm{cw}$ lasers. ${ }^{8-10}$ Data acquisition is made more efficient through time-averaged $\mathrm{cw}$ detection, even with the use of low-grade slow detection systems since the SCRD technique does not rely on the repetition rate and the duration of transient CRD events. Such less-demanding technical configuration for producing and detecting CRD signals should allow an extension of spectral coverage of the CRD technique. Previously, the feasibility of the SCRD technique has been verified in part through the numerical simulation and demonstrated successfully in a preliminary experiment. ${ }^{12}$

In this article, a rigorous theoretical underpinning is presented for the SCRD transmission. The spectral and spatial transfer function of an ideal diffraction lossless cavity with one transverse dimension is derived analytically on the combined basis of ray optics and diffraction theory. Aspects of spatial frequency filtration through a narrow Lorentzian cavity resonance mode clearly accounts for the primary SCRD features including: the exponential decrease in spatial intensity distribution, the ringdown distance (decay constant), the 


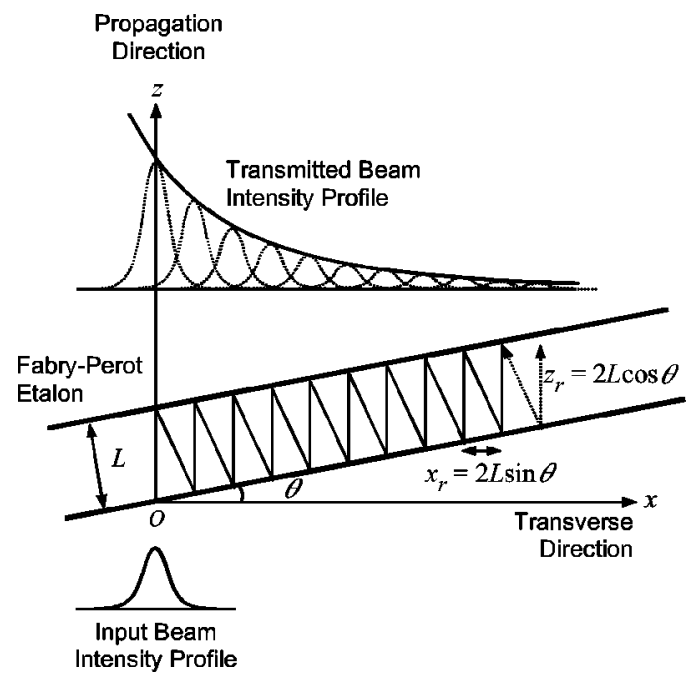

FIG. 1. Optical configuration of a tilted plane Fabry-Perot (PFP) cavity producing spatial cavity ringdown (SCRD) output.

transmitted beam intensity, and the effects of frequency detuning. Theoretical consideration is further extended to the case of nonparallel cavity mirror alignment (wedged PFP cavity) and the deviation of SCRD profiles to nonexponential decays is examined by a numerical simulation. Finally, the theoretical predictions are experimentally confirmed by observing the SCRD signals from a tilted PFP cavity.

It should be mentioned about the theoretical formulation to treat the PFP cavity transmission that our approach taken here is distinctive from the previous ones based on the resonator theory and the modal analysis. ${ }^{13}$ We give focus on the spatial transformation of a transmitted beam. The existing theories that have been developed for the stable cavities comprising spherical mirrors cannot be applicable to PFP cavities with which transverse modes are no longer permitted because of their unstable resonator configuration; any diffracting beam in a PFP cavity blows up in size during cavity round-trips. Even the numerical work done for finite-aperture PFP resonators suffering diffraction $\operatorname{loss}^{14-17}$ is inadequate for our purpose to isolate the physics of spatial filtration due solely to the beam propagation and the interference in a PFP cavity itself. Thus a new theoretical formulation is attempted in this study through a direct integration of the multiple beam interference and the diffraction, excluding the diffraction loss by assuming an ideal PFP cavity with a sufficiently large aperture so as not to truncate the diffracting beams.

\section{THEORETICAL FORMULATION}

An intuitive description of the optical transmission through a tilted PFP cavity can be made readily by a direct account of multiple beam interference between the diffracting beam components in cavity round-trips, where the diffraction theory and the geometrical optics are involved. With the aid of Fourier relation, analytic expression for the cavity transfer function is then derived with respect to both the wavelength (or the optical frequency) and the spatial frequency (or the angular spectrum component) of an input beam. One can thus regard the PFP cavity transmission as the optical filtration that affects both the spectrum and particularly the spatial shape of a transmitted beam while only the spectral transmission function has received much attention so far. The theoretical description will extend to include a wedged PFP cavity transmission. Preserving the essential physics equivalent with that of the previous work based on the waveguide theory, ${ }^{18,19}$ our theoretical formulation is approached in a straightforward fashion without converting the problem into a waveguide geometry. It is also worth remarking that the PFP cavity configuration we consider here includes only a PFP cavity itself for finite-size diffracting beam inputs, which is obviously different from the usual PFP interferometric setup in which a diverging beam source and a focusing lens are incorporated to form ring-shaped Haidinger fringes in the far field.

\section{A. Derivation of PFP cavity transfer function in the SCRD geometry}

Focusing on the essential of a tilted PFP cavity transmission, the theoretical formulation will be worked out for a simplified situation as depicted in Fig. 1. A one-dimensional (1D) beam with its transverse profile along the $x$ coordinate propagates in the positive $z$ direction toward the cavity. Consider a PFP cavity comprising two parallel infinite-plane mirrors with reflectance $R=|\mathcal{R}|^{2}$ and transmittance $T=|\mathcal{T}|^{2}$, separated by the distance $L$. The configuration is made a bit general to cover the SCRD geometry in which the cavity mirror planes are tilted by $\theta$ from the $x$ axis on the $x z$ plane, allowing for the oblique incidence of an input beam equivalently. The PFP transmission process would then involve (i) the intracavity reflections producing multiple transmitted beams with different propagation distances and different lateral walk-offs, (ii) the diffraction of a beam propagating back and forth inside the cavity, and (iii) the multiple beam interference at the cavity exit. ${ }^{20,21}$

Based on the theoretical model combining the geometrical and wave optical aspects aforementioned, the resultant field observable after the exit mirror $E_{\mathrm{FP}}(x ; z)$ is expressible as the sum of all spatially overlapped optical fields leaving the PFP cavity:

$$
E_{\mathrm{FP}}(x ; z)=\sum_{n=0}^{\infty} \mathcal{T}^{2} \mathcal{R}^{2 n} E\left(x-n x_{r} ; z+n z_{r}\right),
$$

where $E(x ; z)$ is the electric field of an input beam, $x_{r}$ $=2 L \sin \theta$ the lateral displacement of a beam at each cavity round trip, $z_{r}=2 L \cos \theta$ the optical path length difference between the consecutive beams, and $n$ the number of roundtrips taken by the field components. The field $E(x ; z)$ is preferentially set to be a plane wave in a given initial intensity profile at $z=0$ where the PFP cavity's entrance mirror is located. Then it undergoes a distortion in both the amplitude profile and the phase front because of the diffraction as the beam propagates over the distance of $z$.

In order to treat the diffraction of a propagating beam, the angular spectrum propagation method ${ }^{22}$ based on the first Rayleigh-Sommerfeld diffraction formula is applied to specify the optical field $E(x ; z)$. The theory is generally valid over the region covering from near to far field. Given the initial field distribution $E\left(x ; z_{0}\right)$ along the $x$ coordinate on a 
particular plane at $z=z_{0}$, the field distribution $E(x ; z)$ at $z$ $>z_{0}$ can be explicitly determined from the transfer equation that is defined in the spatial frequency domain $\left(k_{x}\right)$ as,

$$
\widetilde{E}\left(k_{x} ; z\right)=\left.\widetilde{H}\left(k_{x} ; z-z_{0}\right)\right|_{k} \widetilde{E}\left(k_{x} ; z_{0}\right),
$$

where $\left.\widetilde{H}\left(k_{x} ; z\right)\right|_{k}=\exp \left[i z \sqrt{k^{2}-k_{x}^{2}}\right]$ is the transfer function for free space propagation of a monochromatic light beam with the wave number $k=\omega / c=2 \pi / \lambda$. Here the Fourier transform relation given by

$$
E(x ; z)=\frac{1}{2 \pi} \int d k_{x} \widetilde{E}\left(k_{x} ; z\right) \exp \left[i k_{x} x\right],
$$

is used to directly associate the spatial field distribution $E(x ; z)$ along the $x$ coordinate to the spatial frequency spectrum $\widetilde{E}\left(k_{x} ; z\right)$, which can be interpreted as a plane wave component having the direction cosine vector $(\cos \alpha, \cos \gamma)$ $\equiv\left(k_{x} / k, k_{z} / k\right)$ where $k^{2}=k_{x}^{2}+k_{z}^{2}$. This suggests that a pure diffraction of a beam may be regarded as a linear, dispersive spatial filter of finite bandwidth $\left(k_{x}<k\right)$.

By taking the Fourier transform [Eq. (3)] of the PFP cavity response in Eq. (1) followed by substituting the diffraction formula [Eq. (2)] into it, one could obtain the resultant transmission spectrum $\widetilde{E}_{\mathrm{FP}}\left(k_{x} ; z\right)$ for $z \geqslant z_{0}+L$, relating the input and the output fields simply as

$$
\widetilde{E}_{\mathrm{FP}}\left(k_{x} ; z\right)=\left.\widetilde{H}_{\mathrm{FP}}\left(k_{x} ; z-z_{0}\right)\right|_{k} \widetilde{E}\left(k_{x} ; z_{0}\right),
$$

where $\left.\widetilde{H}_{\mathrm{FP}}\left(k_{x} ; z\right)\right|_{k}$ is found to take the analytic form given by

$$
\left.\widetilde{H}_{\mathrm{FP}}\left(k_{x} ; z\right)\right|_{k}=\frac{\mathcal{T}^{2} \exp \left[i z \sqrt{k^{2}-k_{x}^{2}}\right]}{1-\mathcal{R}^{2} \exp \left[i\left(z_{r} \sqrt{k^{2}-k_{x}^{2}}-x_{r} k_{x}\right)\right]},
$$

where $\mathcal{T}=\sqrt{T} \exp \left[i \varphi_{T}\right]$ and $\mathcal{R}=\sqrt{R} \exp \left[i \varphi_{R}\right]$. The above expression is the transfer function of a PFP cavity for a monochromatic beam of the wave number $k$ with its spatial frequency components at $k_{x}$. The PFP geometry introduces a transfer function $\widetilde{H}_{\mathrm{FP}}$ having peculiar properties: originating from the cavity multiple reflection and the multiple interference other than pure diffraction, the PFP transmission features simultaneous linear spectral $(k=\omega / c)$ and spatial $\left(k_{x}\right)$ filtration within the bandwidth limit, $k_{x}<k$. Unlike the transfer function $\left.\widetilde{H}\left(k_{x} ; z\right)\right|_{k}$ accounting for diffraction only, the filtration done by $\left.\widetilde{H}_{\mathrm{PP}}\left(k_{x} ; z\right)\right|_{k}$ accompanies not only the phase dispersion but also the modulus attenuation of an input beam. It is also noted that the PFP transfer function $\left.\widetilde{H}_{\mathrm{FP}}\left(k_{x} ; z\right)\right|_{k}$ is independent of the choice for the position of a PFP cavity entrance along the $z$ axis between the input beam plane at $z_{0}$ and the observing plane at $z$.

\section{B. Spectral and spatial filtration in a PFP cavity}

Due to the phase factor $\delta$ of the exponential in the denominator of $\widetilde{H}_{\mathrm{FP}}$ in Eq. (5), the PFP transmission $\left|\widetilde{H}_{\mathrm{FP}}\right|^{2}$ in the form of Airy's function imposes a resonance transmission condition given by

$$
\delta=z_{r} \sqrt{k^{2}-k_{x}^{2}}-x_{r} k_{x}+2 \varphi_{R}=2 m \pi,
$$

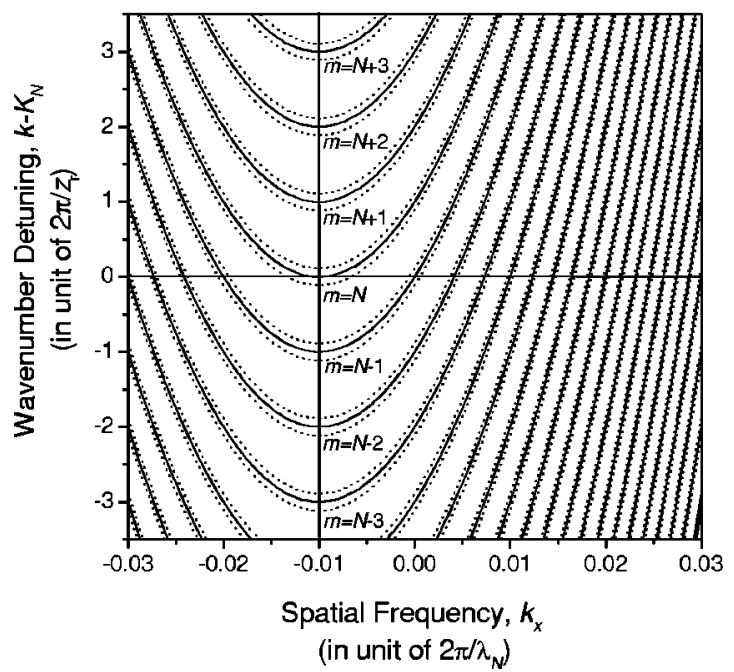

FIG. 2. Spectral and spatial transmission resonance of a tilted PFP cavity with resonance order $m$ 's around $N=20000$ and the tilt angle of $\theta$ $=10 \mathrm{mrad}$. Exact resonance contours satisfying Eq. (6) are represented in solid lines along with adjacent contours of finite detunings $(\Delta m= \pm 0.1)$ in dotted lines.

where $m$ is an integer. According to the resonance condition given by Eq. (6), the resonance transmission contours in the two-dimensional (2D) space of $k$ and $k_{x}$ are thus found to be hyperbolic curves specified by

$$
\frac{k^{2}}{K_{m}^{2}}-\frac{\left(k_{x}+K_{m} \sin \theta\right)^{2}}{\left(K_{m} \cos \theta\right)^{2}}=1,
$$

where we took an abbreviation for $K_{m}=\left(2 \pi m-2 \varphi_{R}\right) / 2 L$. The wave number $K_{m}$ simply implies the $m$ th resonance wave number $K_{m}=2 \pi / \lambda_{m}$ that would hold for an ideal 1D longitudinal Fabry-Perot cavity of length $L$. Spectral $(k)$ and spatial $\left(k_{x}\right)$ transmission windows of a typical PFP cavity are displayed in Fig. 2. Resonant transmission occurs only for input beam components having the wavelength and the spatial frequency that could both fall within the narrow region in the vicinity of such resonance contours. Hyperbolic resonance contours have their apexes periodically positioned at $k=K_{m}$ in the spectral direction $k$ with a period of $2 \pi / z_{r}$ and their symmetric axes shifted by $-K_{m} \sin \theta$ along the direction of spatial frequency $k_{x}$. The width of resonance contours becomes narrower as the finesse $\mathcal{F}=\pi \sqrt{R} /(1-R)$ of a PFP cavity increases. To get an appreciable transmission, the input beam frequency $w=c k$ must be retained at any one of the resonance frequencies within the detuning of $\Omega=\omega-\omega_{(m)}$ less than $\mathrm{FSR} / \mathcal{F}$, with $\mathrm{FSR}=2 \pi c / z_{r}$ denoting the free spectral range of a PFP cavity. ${ }^{23,24}$

A transmission function along spatial frequency $k_{x}$ can be obtained as shown in Fig. 3. Given the frequency $\omega$ $=c k$ of an input beam, the transmission curve is directly the cross section of the contour surface of $\left|\widetilde{H}_{\mathrm{FP}}\right|^{2}$ in Fig. 2, cut along the line in the direction of spatial frequency. For high finesse of a PFP cavity, sharp transmission peaks exist in a series to both sides of a relatively broad transmission window that results from the apex of a hyperbolic resonance contour. The peak width and the separation between adjacent peaks are decreasing as a peak goes away from the broad 

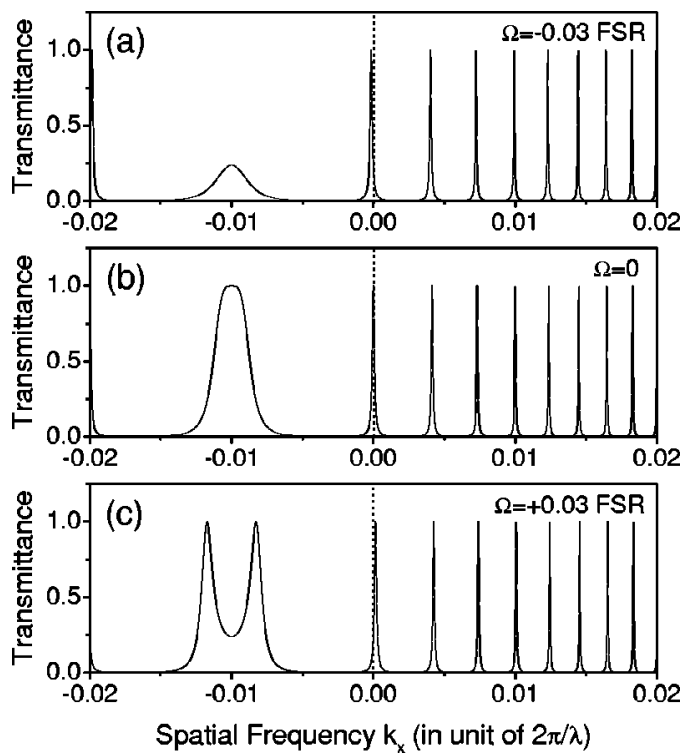

FIG. 3. Tilted PFP cavity transmittance as a function of spatial frequency $\left(k_{x}\right)$ at a given wave number $k$. The transmittance curves are obtained by taking sections of the PFP cavity transfer function along the three constant values of the wave number $k=\omega / c$ with detunings of (a) $\Omega=-0.03$ FSR, (b) $\Omega=0$, and (c) $\Omega=0.03 \mathrm{FSR}$ from the resonance of the order $m$ $=20000$, respectively. A PFP cavity with the mirror reflectivity of $R=0.9$ and the tilt angle of $\theta=10 \mathrm{mrad}$ was considered for the calculation. Note that the location of a sharp transmission peak near $k_{x}=0$ drifts with the detuning.

transmission window. When the input frequency $\omega=c k$ detunes from resonance, the series of peaks moves either outward or inward with reference to the transmission window, depending on the sign of detuning. The width and the separation of peaks changes with the detuning as well. When the detuning becomes an integer multiple of the cavity FSR, the transmission curve is identical with the original. As for the broad transmission window, the effect of detuning is quite different from that for the transmission peaks. With a positive detuning, the transmission window is reduced in the central transmittance but deforms dramatically to produce satellite peaks going outward on both sides. A negative detuning, on the other hand, simply results in a shrunken transmission window with lower transmittance and narrower width. In contrast to the transmission along spatial frequency, the spectral transmission function for a beam component of a given spatial frequency $k_{x}$ is rather plain in its behavior. Transmission peaks of equal width are periodically located with separation of FSR $=2 \pi c / z_{r}$. Depending on the spatial frequency, the periodic transmission peaks simply shift either forward or backward without changing their structure.

In effect to the spatial frequency dependent transmittance clearly shown in Figs. 2 and 3, a transmitted beam is filtered in spatial frequency and thereby deforms in its spatial profile. Such spatial filtration involved would certainly alter the aspects of the PFP cavity transmission that has been usually treated along the longitudinal direction only. ${ }^{25}$ With the higher finesse of a PFP cavity, the resonance transmission structures would become narrower and well isolated from the other adjacent resonance transmission. Mediated by a resonance transmission peak, the transmission will induce a dras- tic spatial filtration especially for an input beam chosen to have a finite incidence angle. This offers a naive physical background of SCRD, which will be discussed in complete detail in the following section.

\section{Physical background of spatial cavity ringdown}

When a PFP cavity is tilted from the normal incidence of an input beam propagating along the $z$ axis, the transmitted output is far more spatially filtered than it would be with the normal incidence. This is because the transmitted beam would see a narrow resonance peak around $k_{x} \sim 0$ in a tilted cavity rather than a relatively broad resonance band for the normal incidence. Since the transmission peak becomes a sharp Lorentzian for a high-finesse cavity, the output beam should have a spatially broadened profile in an exponential decay regardless of the shape of an input beam on the condition that the input beam is sufficiently small in size. Such an exponential decay along the spatial coordinate of a cavity exit will be referred to as spatial cavity ringdown (SCRD) ${ }^{12}$ throughout this article. The time domain analogy of the aspect can be found in the Fabry-Perot cavity response under pulsed laser excitation. ${ }^{26-28}$

Although a crude argument using geometrical optics could support the principle of SCRD to some extent, a complete treatment is only possible on the basis of wave optics, with which both the beam propagation and interference of multiple beams could be taken into account physically. Hence the SCRD concept is established from the transfer function $\left.\widetilde{H}_{\mathrm{FP}}\left(k_{x} ; L\right)\right|_{k}$ we have obtained for a tilted PFP cavity. Resonance transmission in a tilted PFP cavity is permitted for

$$
\left.\widetilde{H}_{\mathrm{FP}}\left(k_{x} ; L\right)\right|_{k}=\frac{\mathcal{T}^{2} \exp \left[i L \sqrt{k^{2}-k_{x}^{2}}\right]}{1-\mathcal{R}^{2} \exp \left[i\left(z_{r} \sqrt{k^{2}-k_{x}^{2}}-x_{r} k_{x}\right)\right]},
$$

taking the phase factor $\delta=z_{r} \sqrt{k^{2}-k_{x}^{2}}-x_{r} k_{x}+2 \varphi_{R}$ that satisfies the $m$ th order resonance condition at $k_{x}=0$ given by

$$
\left.\delta_{0}\right|_{k}=z_{r} k+2 \varphi_{R}=2 m \pi,
$$

with the properly assigned parameter $k=2 \pi / \lambda_{m}$. For high mirror reflectivity $R$ close to 1 , the $\left.\widetilde{H}_{\mathrm{FP}}\left(k_{x} ; L\right)\right|_{k}$ becomes a localized function in the narrow region of $k_{x} \ll 1$ and $\left.\delta\left(k_{x}\right)\right|_{k}-\left.\delta_{0}\right|_{k} \ll 1$, which is true when $\left[\left.\partial \delta\left(k_{x}\right)\right|_{k} / \partial k_{x}\right]_{0} \neq 0$. In the vicinity of the resonance at $k_{x}=0$, the phase factor $\left.\delta\left(k_{x}\right)\right|_{k}$ can be expressible with the first order expansion,

$$
\left.\left.\delta\left(k_{x}\right)\right|_{k} \approx \delta_{0}\right|_{k}+\left[\frac{\left.\partial \delta\left(k_{x}\right)\right|_{k}}{\partial k_{x}}\right]_{0} k_{x} \approx \delta_{0 k}-x_{r} k_{x},
$$

provided that the condition $x_{r} \gg z_{r}\left|k_{x}\right| / k$ is satisfied to make the above linear approximation hold. The condition must be met within the entire region of interest where $\Delta k_{x}$ $\lesssim \Delta \delta_{\text {res }} / x_{r}$ over which the transfer function does not vanish. The condition is thus rewritten cooperatively as $x_{r}^{2}$ $\gg z_{r} \Delta \delta_{\text {res }} / k$ or as, in more practical terms,

$$
\theta_{\text {crit }} \gg \sqrt{\frac{\lambda(1-R)}{2 \pi L}},
$$


by estimating the extent of $\Delta \delta_{\text {res }}$ with $\sim 2 \pi / \mathcal{F}=2 \pi[(1$ $-R) / \pi \sqrt{R}]$. The transfer function can then be approximated near the resonance at $k_{x}=0$ as

$$
\begin{aligned}
\left.\widetilde{H}_{\mathrm{FP}}\left(k_{x} ; L\right)\right|_{k} & \approx \frac{T \exp \left[i\left(k L+2 \varphi_{T}\right)\right]}{1-R \exp \left[-i x_{r} k_{x}\right]} \\
& \approx \frac{T \exp \left[i\left(k L+2 \varphi_{T}\right)\right]}{(1-R)+i R x_{r} k_{x}},
\end{aligned}
$$

and this readily leads to a Lorentzian transmission function for intensity given by

$$
\left|\widetilde{H}_{\mathrm{FP}}\left(k_{x} ; L\right)\right|^{2} \approx \frac{\left(T / x_{r}\right)^{2}}{\frac{1}{4}\left[\frac{2(1-R)}{x_{r}}\right]^{2}+k_{x}^{2}} .
$$

The Lorentzian-shaped transmission in the spatial frequency domain implies, by Fourier transform relation, the transmission output with an exponential decay profile on the spatial coordinate. This is what we call spatial cavity ringdown characterized by a decay constant $s_{d}=x_{r} / 2(1-R)$ which is equal to the inverse of full width at half maximum (FWHM) linewidth $\gamma_{(\mathrm{trm})}=2(1-R) / x_{r}$ of the Lorentzian transmission function.

The generation of SCRD signals through spatial filtration might be invoked alternatively from the argument based on geometrical optics. From Eq. (1) we could roughly expect a ringdown intensity profile along the spatial coordinate $x$ on the condition that the influence of diffraction does not seriously alter the physics involved. A tilted PFP cavity functions so as to produce the lateral displacement and the intensity drop of consecutively outgoing multiple beams as depicted in Fig. 1. The intensity of an outgoing beam component after $n$ round trips diminishes as $I \propto I_{o} R^{2 n} \approx I_{o}$ $\times \exp [-2 n(1-R)]$ and the laterally walks off by $x=n x_{r}$ $=2 n L \sin \theta$. On this account, the cavity output will follow the spatial dependence of $\exp \left[-2(1-R) x / x_{r}\right]$ and the decay constant $s_{d}$ of such profile can be readily predicted as

$$
s_{d}=\frac{x_{r}}{2(1-R)}=\frac{L \sin \theta}{1-R}
$$

which will be called ringdown distance hereafter.

\section{Theoretical treatment for a misaligned PFP cavity with nonparallel mirrors}

Nonparallelism of the cavity mirror alignment ${ }^{29,30}$ is one of the common experimental defects likely to occur in practice and might give rise to much difference between the theoretical expectations made for an ideal PFP cavity.

The convenience of a transfer function in terms of spatial frequency is, however, no more available with such nonparallel Fabry-Perot cavities. Such a transfer function does not exist owing to the fact that a non parallel PFP cavity would successively produce the outgoing beams with a spatial frequency being deviated from the initial spatial frequency component with the increasing number of cavity reflections $n$. As illustrated in Fig. 4, the propagating beams would undergo wave front tilts by $2 n \varepsilon$ and extend its dimension on a specific $z$ plane by the factor of $1 / \cos 2 n \varepsilon$ when the

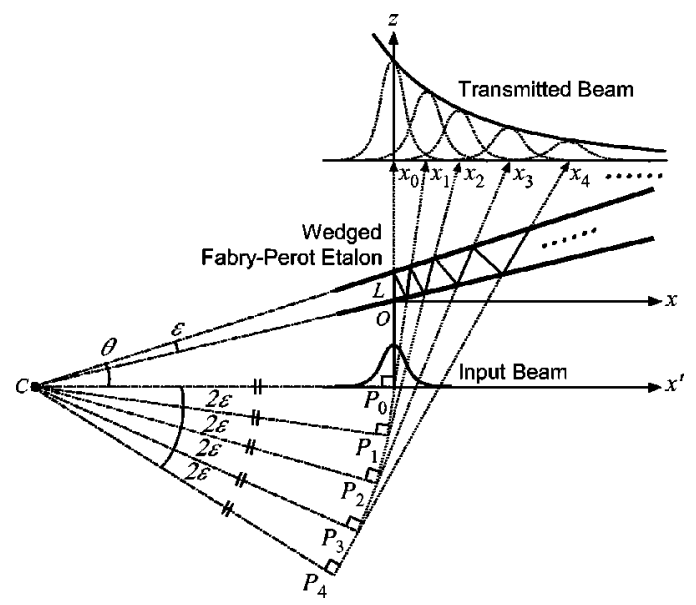

FIG. 4. Optical geometry of a tilted PFP cavity with nonparallel mirror alignment.

planes of cavity mirrors are wedged by $\varepsilon$. The lateral profile shift on the $x$ coordinate and the phase delay between adjacent outgoing beams are therefore no longer preserved as constants. The nonstationary condition exerted on the spatial features along the lateral direction does not allow the spatial frequency arguments to be valid.

Lacking in any transfer function for a nonparallel PFP cavity, we still have a way to deal with the problem. By taking advantage of the theoretical formulation developed in the spatial domain, we can resort to the numerical technique. Recalling that our approach combines the geometrical and the wave optical aspects of a PFP cavity, the nonparallel PFP cavity can be numerically investigated based on the formulation modified from Eq. (1) as

$$
E_{\mathrm{FP}}(x ; L)=\sum_{n=0}^{\infty} \mathcal{T}^{2} \mathcal{R}^{2 n} E_{n}\left(x-x_{n} ; L+z_{n}\right),
$$

where $E_{n}(x ; z)$ is the diffracting field of a propagating beam at $z$ imposed with a wave front tilt by the angle of $2 n \varepsilon, x_{n}$ the lateral shift of the $n$th outgoing beam with reference to the input position, $z_{n}$ the optical path length of the $n$th outgoing beam with reference to the cavity entrance, and $n$ the number of round-trips of a propagating beam. As in Fig. 4, illustrating the wedged PFP cavity with a virtual pivot point of extended mirror planes that is introduced for easier geometrical calculations, ${ }^{31,32}$ the lateral profile shift $x_{n}$ at $z=L$ is obtained analytically as

$$
x_{n}=\frac{L}{\tan \varepsilon}\left[\frac{\cos \theta}{\cos (\theta+2 n \varepsilon)}-1\right] \text {, }
$$

which is approximately $2 n L(\theta+n \varepsilon)$ for small angles, and the propagation distance $z_{n}$ at $z=L$ is derived to be

$$
z_{n}=L\left[\frac{\cos \theta \tan (\theta+2 n \varepsilon)}{\tan \varepsilon}-\left(\frac{\sin \theta}{\tan \varepsilon}-\frac{1}{\cos \theta}\right)-1\right],
$$

which can be reduced to $2 n L\left(1+n \theta \varepsilon+4 n^{2} \varepsilon^{2} / 3\right)$ in a small angle approximation. 


\section{NUMERICAL INVESTIGATION OF SPATIAL CAVITY RINGDOWN: PFP CAVITY TRANSMISSION SIGNALS WITH OBLIQUE BEAM INCIDENCE}

\section{A. Basic spatial and spectral features}

Typical ringdown signal profiles were simulated in the spatial domain by the direct numerical calculation of $E_{\mathrm{FP}}(x ; L)=\sum_{n=0}^{\infty} \mathcal{T}^{2} \mathcal{R}^{2 n} E\left(x-n x_{r} ; L+n z_{r}\right)$ presented in Eq. (1). The diffracting optical field $E(x ; z)$ was numerically calculated by using the first Rayleigh-Sommerfeld diffraction formula, given the initial transverse beam profile of a monochromatic input at $z=0$. For the following numerical simulation, a simple model for the diffracting beam inputs was chosen with a monochromatic super-Gaussian beam $\left.E(x ; z)\right|_{k}$ in the form of

$$
E(x ; z)=A(x ; z) \exp \left[-\frac{\ln 2}{2}\left(\frac{x}{w_{\mathrm{hw}}}\right)^{2 q}\right] \exp [i k z],
$$

where $w_{\mathrm{hw}}=D / 2$ is the half width of an input beam, $q$ the order of super-Gaussian, $k=\omega / c$ the wave number, and $A(x ; z)$ the complex amplitude variation caused by the diffraction of a propagating beam. The input beams are assumed to locate its waist at the entrance face of the PFP interferometer at $z=0$ by letting $A\left(x ; z_{0}\right)=0$. Note that one might alternatively use the analytic solution $E(x ; z)$ in the spatial domain for a Gaussian beam $(q=1)$. For higher order super-Gaussian beams $(q>1)$, one can resort to the numerical calculation for both $E(x ; z)$ and $\widetilde{E}\left(k_{x} ; z\right)$ based on the diffraction formula given by Eqs. (2) and (3). However, most of our analytic discussion in this article will be made with a Gaussian $(q=1)$ input whose spatial frequency spectrum $\left|\widetilde{E}\left(k_{x} ; z_{0}\right)\right|^{2}$ is easily available as $\left|\widetilde{E}_{0}\right|^{2} \exp \left[-\left(D^{2} / 4 \ln 2\right) k_{x}^{2}\right]$.

In fact, further constraints must be satisfied for SCRD:

$$
\gamma_{(\operatorname{trm})} \ll \Delta k_{x(\text { in })} \ll \Gamma_{(\operatorname{trm})},
$$

(i) the linewidth $\gamma_{(\mathrm{trm})}=2(1-R) / x_{r}$ of a Lorentzian transmission peak must be much narrower than the spatial frequency extent $\Delta k_{x(\mathrm{in})}=4 \ln 2 / D$ of an input beam, generating a genuine exponential decay signal regardless of the shape of an input beam, and (ii) the spacing between the adjacent transmission peak of next order $\Gamma_{(\operatorname{trm})}=\sqrt{k^{2} \sin ^{2} \theta+k(2 \pi / L)}$ $-k \sin \theta$ must be marginally wider in order for an input beam not to be resonant with more than one transmission peak, otherwise multiple exponential decays accompanying spiky interference fringes will result. The constraint specified in Eq. (19), would readily yield the relations between the cavity tilt angle $\theta_{\mathrm{SCRD}}$ and the input beam size $D$ given by

$$
\frac{1-R}{4 \ln 2}\left(\frac{D}{L}\right) \ll \sin \theta_{\mathrm{SCRD}} \ll \frac{\pi}{4 \ln 2}\left(\frac{D}{L}\right) .
$$

\section{Exponential decay profiles}

Figure 5 shows the results obtained for the intensity profiles of the SCRD signal generated using a detuned Gaussian $(q=1)$ beam input and with the following simulation parameters: cavity length $L$ of $10 \mathrm{~mm}$, mirror reflectance $R$ of 0.99 , cavity tilt $\theta$ of $5 \mathrm{mrad}$, input beam wavelength $\lambda$ at $632.8 \mathrm{~nm}$, and initial beam size $D$ of $1 \mathrm{~mm}$ in FWHM. Using Eq. (5) the cavity transmission curves are also plotted as a
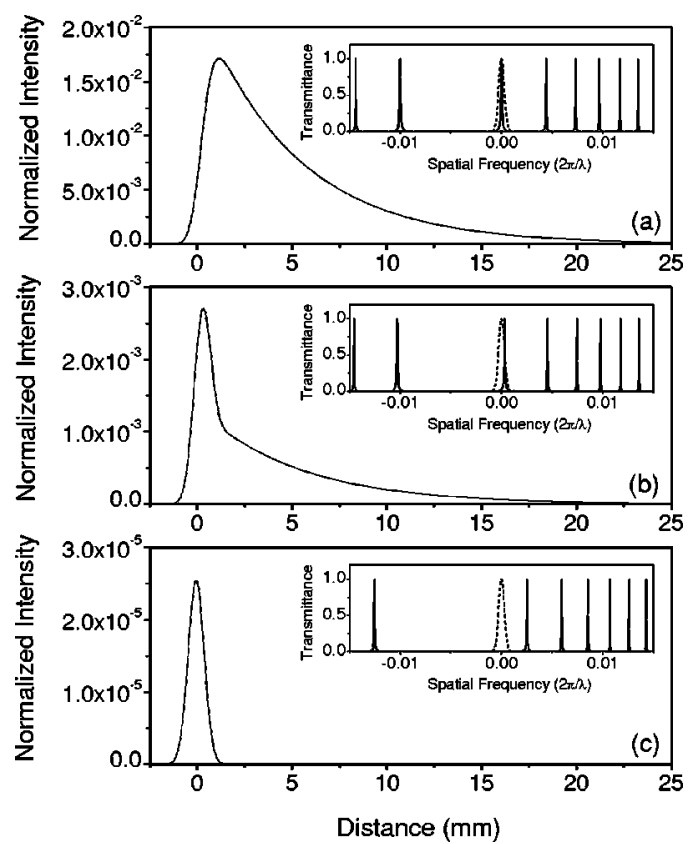

FIG. 5. SCRD signal generation in a tilted PFP cavity by using Gaussian beam inputs with frequency detunings. The transmitted spatial profiles are shown for the frequency detunings of (a) $\Omega=0$, (b) $\Omega=0.05 \mathrm{FSR}$, and (c) $\Omega=0.5$ FSR. In the inset of each figure the transmission curve of the tilted PFP cavity (solid) and the input beam distribution (dotted) are depicted as a function of spatial frequency. The results shown here are for a 10-mm-long PFP cavity comprising mirrors of the reflectivity $R=0.99$ with the cavity tilt angle of $\theta=5 \mathrm{mrad}$, illuminated by a Gaussian beam input at the wavelength of $632.8 \mathrm{~nm}$ having the FWHM width of $D=1.0 \mathrm{~mm}$

function of spatial frequency for each detuned input beam. The input beam wave number $k=2 \pi / \lambda$ was reassigned to the nearest resonance wave number $k_{(m)}=2 \pi / \lambda_{(m)}$ so that the dc spatial frequency component $\left(k_{x}=0\right)$ of an input beam can be resonant with a tilted cavity by satisfying the condition $k_{(m)} z_{r}+2 \varphi_{R}=2 \pi m$ where $\mathcal{R}=\sqrt{R} \exp \left(i \varphi_{R}\right)$ and an integer $m$. Then the frequency detuning $\Omega$ of the beam was defined by $\Omega=\omega-\omega_{(m)}$ with $\omega_{(m)}=c k_{(m)}$.

As shown in the each inset of Fig. 5, the input beam distribution (dotted) is centered at dc spatial frequency $\left(k_{x}\right.$ $=0$ ) and has a finite spatial frequency extent in the Gaussian shape which is inversely proportional to the width of the input beam. On the other hand, the center of the spatial frequency transmission curve (solid) is shifted from $k_{x}=0$ in the presence of cavity tilt $\theta$ and locates at $k_{x}$ $=-0.005[2 \pi / \lambda]$ where a broad transmission band is supposed to appear and disappear with the detuning. Instead, the first transmission peak is positioned at dc spatial frequency ( $k_{x}=0$ ) in the case of zero detuning $\Omega=0$. As the detuning increases, the transmission peaks move outward from the center and this in turn gives rise to the change in the overlap feature between the input beam spatial frequency distribution and the first transmission peak.

It is notable that the consequence of multiple beam interference of diffracting beams in a PFP etalon does not play a serious role in the exponential decay feature which is expected roughly from Eq. (1); despite the diffractive distortion occurring at each individual outgoing beam component in intensity and phase profile, a smooth decay of a SCRD signal 
is obtained if the input beam diameter is greater than $x_{r}$. The decay has been verified to be a single exponential decay through a numerical curve fit when the input beam is resonant with the tilted cavity. As the input frequency is detuned from the cavity resonance, the onset intensity of a decay part decreases and a transient peak begins to manifest at the signal forefront. Still the decay envelope following the transient peak is an exponential decay but with a ringdown distance deviated from that in case of resonance. When the detuning goes beyond a certain threshold toward antiresonance, a transmitted signal merely contains a diminished transient peak without any decaying part.

Since the SCRD profile is directly the Fourier transform of the transmitted spatial frequency spectrum, the SCRD features can be inferred in the context of how the cavity transmission curve overlaps with the spatial frequency distribution of an input beam. When the transmission peak locates exactly at the center of the spatial spectrum of an input [see Fig. 5(a)], a smooth exponential decay signal is obtained with maximum signal. With a frequency detuning [see Fig. 5(b)], the cavity transmission is attributed by the Lorentzian peak transmission producing an exponential decay signal and the residual transmission permitting a transient peak profile at the signal front. Because of the reduced coupling of spatial frequency components through the Lorentzian peak, the intensity level of the decaying part is lower than that in the case of resonance. The transient peak is the consequence of a whole spatial spectrum transmission of an input beam that is achieved by the nearly flat residual transmittance of a cavity spanning over the entire spatial frequency region. Above a certain frequency detuning [see Fig. 5(c)], only a small transient peak profile remains because of the absence of the contribution from the Lorentzian peak transmission.

\section{Spatial and spectral behaviors with the frequency detuning}

Examining the ringdown signal profiles, the variation in ringdown distance $s_{d}$ and ringdown signal intensity was quantitatively assessed as changing the frequency detuning $\Omega$ as shown Fig. 6. In the presence of detuning, the ringdown distance reveals a deviation from the theoretical prediction $s_{d}=L \sin \theta /(1-R)$ which has been deduced on the basis of geometrical optics and found to be identical with the result of wave optics in the first order approximation. The discrepancy is found to be proportional with the frequency detuning as long as a ringdown signal contains a part that exponentially decays. The reason for this detuning dependence of ringdown distance can be inferred by the transmission peak structure of a detuned cavity and the finite spatial frequency distribution of an input beam. By using Eqs. (9) and (10), the transmission function for a frequency detuning $\Omega$ $=\Omega_{\mathrm{FSR}}[\mathrm{FSR}]$ in terms of $\mathrm{FSR}=2 \pi c / z_{r}$ can be derived from modifying Eq. (13) to read

$$
\left|\widetilde{H}_{\mathrm{FP}}\left(k_{x} ; L\right)\right|^{2} \approx \frac{\left(T / x_{r(\Omega)}\right)^{2}}{\frac{1}{4}\left[\frac{2(1-R)}{x_{r(\Omega)}}\right]^{2}+\left(k_{x}-k_{x(\Omega)}\right)^{2}},
$$
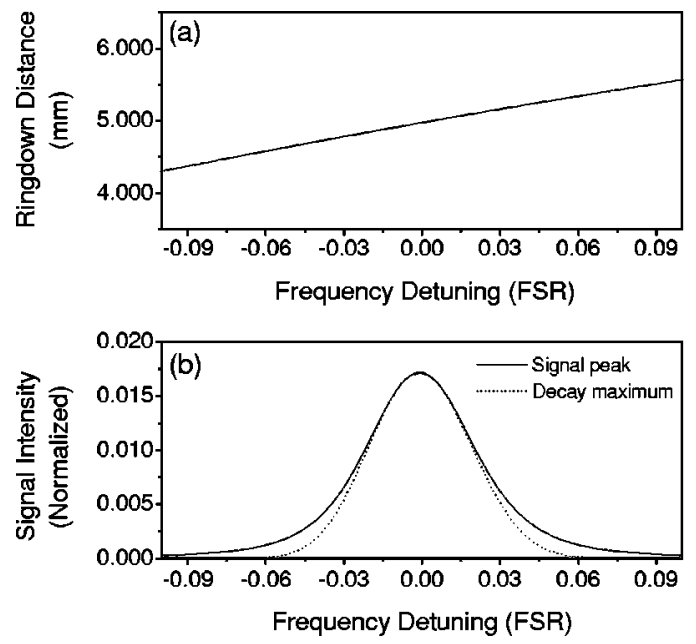

FIG. 6. SCRD behaviors with the frequency detuning $\Omega$ between a cavity resonance and an input beam. The dependence is displayed for (a) the ringdown distance $s_{d}$ and (b) the characteristic intensity levels of a SCRD signal where the solid line represents the peak intensity of a SCRD signal and the dotted line the onset intensity of an exponential decay envelope. The results shown here are for a 10-mm-long PFP cavity comprising mirrors of the reflectivity $R=0.99$ with a cavity tilt angle of $\theta=5 \mathrm{mrad}$, illuminated by a Gaussian beam input at the wavelength of $632.8 \mathrm{~nm}$ having the FWHM width of $D=1.0 \mathrm{~mm}$.

where the effective lateral shift $x_{r(\Omega)}=x_{r}+\lambda \Omega_{\mathrm{FSR}} / \sin \theta$ and the resonance spatial frequency $k_{x(\Omega)}=2 \pi \Omega_{\mathrm{FSR}} / x_{r}$ have been found.

For positive (negative) detuning, the transmission peak shifts to higher (lower) spatial frequency and the peak width gets narrowed (broadened). Within the extent of the spatial frequency distribution of an input beam, a shifted transmission peak still permits a ringdown signal but with the ringdown distance which is different from that at exact resonance owing to the change in the transmission peak width. The analytic prediction for such ringdown distance variation can be obtained directly from the resonance width of the detuned transmission peak given in Eq. (21), which leads to

$$
s_{d(\Omega)}=\frac{L \sin \theta}{(1-R)}\left[1+\left(\frac{\lambda}{2 L \sin ^{2} \theta}\right) \Omega_{\mathrm{FSR}}\right] .
$$

An alternative explanation can be made with terms a bit more geometrical: a finite-size beam having a geometrical incidence angle $\theta$ actually consists of spatial frequency components distributed within a certain extent, or equivalently, beam components of different propagation directions around the incidence angle $\theta$. The detuned cavity is then more resonant with a beam component at a different incidence angle rather than that at the original incidence angle $\theta$. Thus the beam component at an incidence angle of $\theta^{\prime}$ is selectively transmitted to result in a ringdown distance of $s_{d}^{\prime}$ $=L \sin \theta^{\prime} /(1-R)$.

The detuning-dependent ringdown signal takes place in a certain range of frequency detuning as shown in Fig. 6(b), which is determined primarily by the size of an input beam $D$, or alternatively the spatial frequency extent $\Delta k_{x(\text { in) }}$. The resonance interval of $\Omega_{\mathrm{FSR}}$ can be estimated from $\Delta k_{x(\mathrm{in})}$ $=k_{x(\Omega)}=2 \pi \Omega_{\mathrm{FSR}} / x_{r}$, which reads as 


$$
\Delta \Omega=\frac{4 \ln 2}{\pi} \frac{L \sin \theta}{D}[\mathrm{FSR}] .
$$

Another important feature to be noted is the redshift of the resonance center. As implied by Eq. (21), the Lorentzian transmission peak becomes broader in linewidth for the negative detuning, allowing more coupling of the spatial frequency components of an input for transmission. The tradeoff between the widening of the transmission window, $\gamma_{(\mathrm{trm})}=2(1-R) /\left[x_{r}+\lambda \Omega_{\mathrm{FSR}} / \sin \theta\right]$, and the decrease in the intensity of spatial frequency components, $I_{k_{x}(\mathrm{in})} \propto$ $\exp \left[-\left(D^{2} / 4 \ln 2\right)\left(2 \pi \Omega_{\mathrm{FSR}} / x_{r}\right)^{2}\right]$ with negative detunings, determines the resonance peak detuning to appear at

$$
\begin{aligned}
\Omega_{c} & =-\frac{L \sin ^{2} \theta}{\lambda}\left[1-\sqrt{1-\frac{2 \ln 2}{\pi^{2} \sin ^{2} \theta} \frac{\lambda^{2}}{D^{2}}}\right][\mathrm{FSR}] \\
& \approx-\frac{\ln 2}{\pi^{2}}\left(\frac{\lambda L}{D^{2}}\right)[\mathrm{FSR}] \quad \text { for } \lambda \ll D \sin \theta,
\end{aligned}
$$

where the dependence on the cavity tilt angle $\theta$ could be eliminated for the large angle regime.

\section{Potential for spectroscopic applications}

Further numerical calculation has verified that the simple analytic prediction for ringdown distance $s_{d_{0}}=L \sin \theta /(1$ $-R$ ) is valid over a wide choice for cavity length $L$, per-pass loss of a cavity $\mathcal{L}=(1-R) \ll 1$, incidence angle $\theta \ll 1$, and input beam parameters including its shape $q$ and size $D$. On this ground, any change in the ringdown distance of a SCRD signal can be exploited to probe additional cavity loss $\mathcal{L}_{\text {sample }}=\alpha L$ that is to be introduced between the cavity mirrors as a sample gas with absorption coefficient $\alpha$. Known all other system parameters $L, \theta, R$, and $\omega$ the ringdown distance in the presence of the sample would become $s_{d}$ $=(L \sin \theta) /[(1-R)+\alpha L]$, and thereby the sample loss $\mathcal{L}_{\text {sample }}$ can be determined from $\alpha L=L \sin \theta\left(s_{d}^{-1}-s_{d_{0}}^{-1}\right)$.

In the experimental point of view, one is to observe the cavity output signals on resonance characterized more likely by maximum signal levels rather than by a theoretical assignment. Therefore the realistic ringdown distance would be observed as

$$
s_{d\left(\Omega_{c}\right)}=\frac{L \sin \theta}{(1-R)}\left[1-\frac{\ln 2}{2 \pi^{2}}\left(\frac{\lambda}{D \sin \theta}\right)^{2}\right]
$$

by substituting Eq. (25) into Eq. (22). This would cause a fractional accuracy error involving the cavity loss measurement by the factor of $\left(\ln 2 / 2 \pi^{2}\right)(\lambda / D \sin \theta)^{2}$, but can be made negligible in the experiment. Even worse ambiguity may arise when one could just maintain the cavity resonance no better than the detuning $\Omega_{\mathrm{err}}=\eta_{\mathrm{err}} \Delta \Omega$, which would limit the relative precision of the ringdown distance measurement $\sigma_{s} / s_{d}$ as

$$
\frac{\sigma_{s}}{s_{d}}=\frac{2 \ln 2}{\pi}\left(\frac{\lambda}{D \sin \theta}\right) \eta_{\mathrm{err}} .
$$

The higher signal-to-noise (S/N) ratio of SCRD profiles and the tighter frequency control are therefore the indispensable requirements to promise for the higher precision measure-
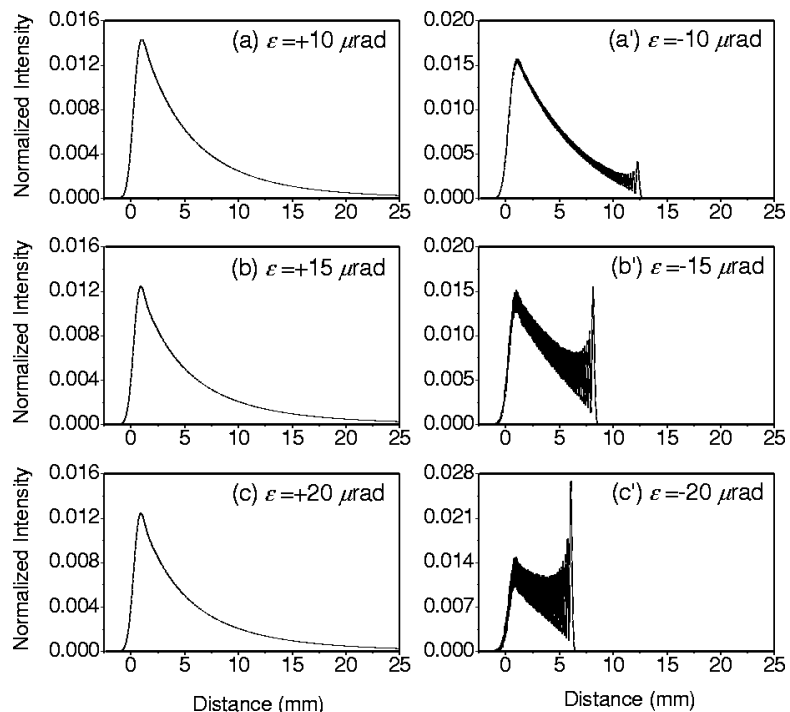

FIG. 7. SCRD signals from wedged PFP cavities using a small-size input beam. On the left column are the SCRD signals for the positive wedge angles of (a) $10 \mu \mathrm{rad}$, (b) $15 \mu \mathrm{rad}$, and (c) $20 \mu \mathrm{rad}$, and on the right column the negative wedge angles of (a') $-10 \mu \mathrm{rad},\left(\mathrm{b}^{\prime}\right)-15 \mu \mathrm{rad}$, and (c') $-20 \mu \mathrm{rad}$. The results shown here are for a 10-mm-long PFP cavity comprising mirrors of the reflectivity $R=0.99$ with a cavity tilt angle of $\theta$ $=5 \mathrm{mrad}$, illuminated by a Gaussian beam input at the wavelength of 632.8 $\mathrm{nm}$ having the FWHM width of $D=1.0 \mathrm{~mm}$.

ment of the ringdown distance $s_{d}$, and the higher sensitivity on the cavity loss $\mathcal{L}$ in turn, from the minimum detectable loss given by $\mathcal{L}_{\min }=(1-R) \sigma_{s} / s_{d}$.

\section{B. Spatial cavity ringdown in a wedged PFP cavity}

\section{Dependence on the mirror wedge angle}

From the viewpoint of SCRD signal generation, the numerical investigation was made on how the nonparallelism of PFP cavity mirrors alters the ringdown behaviors found with an ideal tilted PFP cavity. The output signals from a wedged PFP cavity using a small input beam were calculated for several cavity wedge angles and the results are shown in Fig. 7. The PFP cavity and the input beam are presumed to be resonant $(\Omega=0)$ at the center of the beam incidence and the size of an input beam $D=1 \mathrm{~mm}$ was set to be sufficiently smaller than the ringdown distance $s_{d}=5 \mathrm{~mm}$.

For positive wedge angles with which the cavity gap becomes larger in the direction of ringdown, the ringdown signals show little change in the eye but only a slight increase of the ringdown distance and a nonexponential decay envelope. In contrast, negative wedge angles cause a decrease in the ringdown distance accompanying nonexponential transmission, which is nearly proportional to the magnitude of wedge angles. The most intriguing feature present in this case is that the nonexponential decay envelop abruptly stops at a certain position and the intensity modulation is superimposed on the decay envelope. As can be easily expected, the signal envelope decreases more rapidly with the larger magnitude of negative wedge angle. It is inferred that the negative wedge angle gradually reduces the inclination angle of multiply reflected beams and finally produces counterpropagating wave components after a certain number of 
reflections to interfere with the wave components in the original direction, which gives rise to partial standing waves.

It would be instructive to make a crude prediction for the transmitted intensity distribution $I(x)$ : this can be obtained from the geometrical argument that

$$
I_{n} \propto \frac{R^{2 n}}{(d x / d n)} \approx \frac{\exp [-2 n(1-R)]}{(d x / d n)},
$$

using the relations deduced from Eq. (16), $n \approx x / 2 L \theta$ $-(\varepsilon / \theta)(x / 2 L \theta)^{2}$ and $d x / d n=2 L \theta+4 L \varepsilon n$. The transmitted intensity then follows the spatial profile in the form of

$$
I(x) \approx \frac{I_{o} \exp [-x / S(x)]}{V(x)},
$$

with the nonexponential correction functions given by

$$
\begin{aligned}
& S(x)=\frac{L \theta}{1-R}\left(1-\frac{1}{2} \kappa x\right), \\
& V(x)=1+\kappa x-\frac{1}{2} \kappa^{2} x^{2},
\end{aligned}
$$

where $\kappa=\varepsilon / L \theta^{2}$ for $\varepsilon \ll \theta \ll 1$.

In addition, a steep break point in the decay profile occurring for the case of a negative wedge can be estimated roughly as well. What is supposed to happen at such a location is the turning of the pointing direction of a transmitted field to have the $x$-component increment $\left(x_{n}-x_{n-1}\right)$ of its exit position be reversed from positive to negative values; the condition $(d x / d n)=0$ readily dictates the break point to be present at

$$
s_{\text {brk }}=L \theta^{2} / 2|\varepsilon|
$$

regardless of the mirror reflectivity $R$. At the tuning point, the spatial dispersion proportional to $(d x / d n)$ takes on a minimum and the counterpropagating waves are most likely to be comparable with each other in the intensity, thus resulting in the peaking of intensity and the maximum modulation contrast. The theoretical expectation for the break points on the condition in Figs. 7(a')-7( $\left.\mathrm{c}^{\prime}\right)$, yields, respectively, 12.5, 8.3 , and $6.25 \mathrm{~mm}$, which is in good accordance with the result of direct numerical calculation. The modulation has the smaller period at the position closer to the onset of a decay signal since the counterpropagating wave components could have larger intersection angles than those near the turning point.

\section{Influence of the transverse extent of an input beam}

Transmission in a wedged PFP cavity with the ringdown geometry also exhibits another kind of intensity modulation other than that caused by the counterpropagating wave interference. It is originated from the nonlinear increase (decrease) of phase delays in the multiply reflecting beam components because of the increasing (decreasing) optical path length per cavity round-trip in a positively (negatively) wedged cavity. This can be understood from Eq. (17), which approximates to the nonlinear increase of path length $z_{n}$ $=2 n L\left(1+n \theta \varepsilon+4 n^{2} \varepsilon^{2} / 3\right)$. The resultant vector sum of the complex amplitude of total multiple beams produces inten-
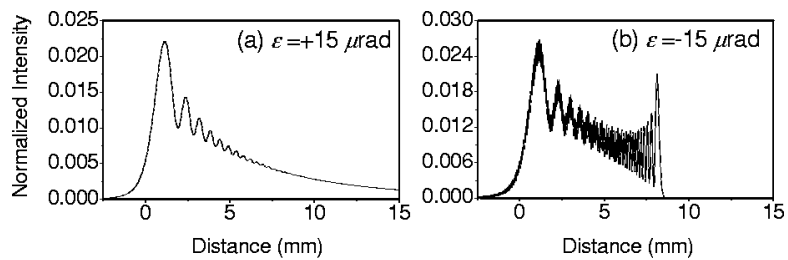

FIG. 8. SCRD signals from wedged PFP cavities using a large-size input beam. The results are shown for the input beam having the FWHM width of $D=6 \mathrm{~mm}$ and with the cavity wedging angle $\varepsilon$ of (a) $15 \mu \mathrm{rad}$ and (b) $-15 \mu \mathrm{rad}$. The results shown here are for a $10-\mathrm{mm}$-long PFP cavity comprising mirrors of the reflectivity $R=0.99$ with a cavity tilt angle of $\theta$ $=5 \mathrm{mrad}$.

sity maxima and minima alternating with periods that become shorter gradually in the latter part of ringdown profiles. The intensity modulation could extend over a distance that is spanned by the spatial dimension of an input beam. ${ }^{33-37}$

Spatial ringdown signals from a wedged PFP cavity were calculated with a large-size $(D=6 \mathrm{~mm})$ input beam which has a dimension comparable with the ringdown distance $\left(s_{d} \sim 5 \mathrm{~mm}\right)$. Figure 8 clearly shows the intensity modulation that could occur not only by the wedging of cavity mirrors but also by the large dimension an input beam. Further numerical calculation confirmed that the modulation takes place within the extent of the order of an input beam span and the modulation visibility gets higher when the number of interfering beams is made larger by enlarging the input beam size. Also found is that the modulation periods become shorter for a larger wedge angle of the cavity. Comparing the profiles shown in Figs. 7 and 8, one can note that the modulation of this kind is additionally superposed on the ringdown signal envelope along with the typical features in a wedged PFP cavity.

\section{Frequency detuning behavior}

Intriguingly, the effects of frequency detuning on the ringdown signal profile in a wedged PFP cavity are more asymmetric with the sign of detuning as compared with those in an ideal PFP cavity. Spatial ringdown signals from a wedged PFP cavity are shown in Fig. 9 in the presence of frequency detunings. First, let us examine the detuning behavior in a positively wedged cavity. If frequency detuning is positive, the change in the profile of a ringdown signal with the increase of detuning is similar to that which occurred with an ideal PFP cavity. The intensity level of a decay signal is diminished and a transient peak appears at the forefront of the ringdown profile in the presence of detuning. With negative detuning, however, the transient peak does not manifest until reaching a certain threshold magnitude of detuning and thus the asymmetry in the detuning behavior is implied by comparing with the case of positive detuning in the same magnitude. The absence of a transient peak can be understood by the reasoning that a negatively detuned input beam is not resonant with a wedged PFP cavity at the position of beam incidence but becomes resonant gradually by the lateral shift of a multiply reflected beam position where the increased cavity gap is more favorable for the lower input beam frequency. As a consequence, the maximum position of 

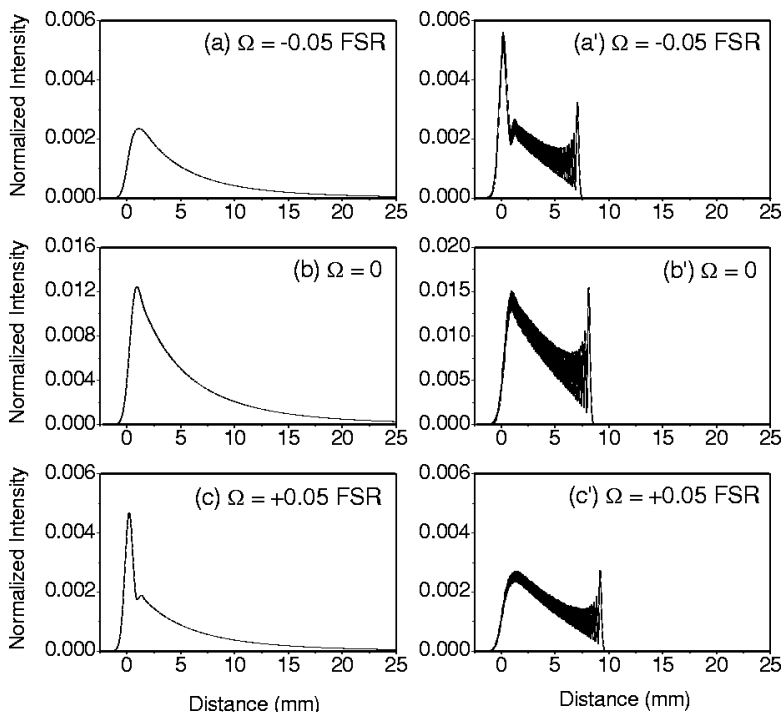

FIG. 9. SCRD signals from wedged PFP cavities with different frequency detunings. The plots in the left column are with the wedge angle of $\varepsilon$ $=15 \mu \mathrm{rad}$ and the detunings of (a) $-0.1 \mathrm{FSR}$, (b) 0 , and (c) 0.1 FSR. The plots in the right column are with the wedge angle of $\varepsilon=-15 \mu \mathrm{rad}$ and the detunings of (a') -0.1 FSR, (b') 0, and (c') 0.1 FSR. The results shown here are for a 10-mm-long PFP cavity comprising mirrors of the reflectivity $R=0.99$ with a cavity tilt angle of $\theta=5 \mathrm{mrad}$, illuminated by a Gaussian beam input at the wavelength of $632.8 \mathrm{~nm}$ having the FWHM width of $D$ $=1.0 \mathrm{~mm}$. Note that the behaviors with frequency detunings are reversed for the two wedge angles of opposite sign.

a negatively detuned signal is shifted toward the latter part and the shape of the peak becomes dull without a sharp transient peak. Starting from a positive frequency detuning, in contrast, the input beam could never have a chance to reduce the frequency detuning between the cavity. One should also note that the behavior of detuning is the reverse of each other for the positive and the negative wedge PFP cavities. The reason can be easily deduced by reminding one of the facts that the resonance conditions to be met with an input beam frequency are opposite in the two cases along the lateral direction

\section{EXPERIMENTAL DEMONSTRATION OF SPATIAL CAVITY RINGDOWN}

\section{A. Experimental setup}

Optical transmission signals from a tilted PFP cavity were observed experimentally and then compared with the results of numerical simulation. This could provide a direct verification of the theoretical footing made in this article for the SCRD transmission. In addition, the experiments were also included for a wedged PFP cavity transmission, with a practical concern that the nonparallel cavity mirror misalignment is one of the most common defects in a PFP cavity that might be seriously detrimental to the transmission characteristics.

In the experimental demonstration, a PFP etalon was constructed with two plane mirrors $(R \sim 0.99,25.4-\mathrm{mm}$ diameter) separated by $L=10 \mathrm{~mm}$, on a commercial SuperInvar interferometer mount (Burleigh, RC110). A cw He-Ne laser (Spectra Physics, 117A) stabilized within about $1 \mathrm{MHz}$ was used as a light source at the wavelength of $632.8 \mathrm{~nm}$. The $s$-polarized laser beam was regulated in power to have less than $100 \mu \mathrm{W}$ and transformed through a spatial filtering telescope to permit a well-defined Gaussian profile with its beam waist in the diameter of $\sim 0.5 \mathrm{~mm}$ at the cavity entrance. To accomplish a SCRD geometry, we began the optical alignment by directing the input laser beam toward the PFP cavity in normal incidence and then adjusting the cavity mirrors to be parallel with each other, which was confirmed by the circular symmetry of a transmitted beam profile. Finally, the input beam was slightly steered away from the normal incidence by a known amount of tilt angle $\theta$ $=\sin ^{-1}(d / l)$ which was specified from the deviation of a reflecting beam position $d$ on the screen placed in the distance of $l=10 \mathrm{~m}$ away from the entrance mirror. Throughout the entire experiment, the PFP cavity length $L$ needs to be fine controlled in order to either maintain resonance or impose a desired detuning with reference to the input laser frequency. The detuning $\Omega$ between the PFP cavity resonance and the input laser frequency was achieved equivalently by changing the cavity resonance frequency, instead of laser frequency adjustment. The procedure was done by biasing a control voltage on a hollow cylindrical piezoelectric transducer (PZT) where the cavity exit mirror attached on. The transmitted spatial beam profiles were digitized by a highdefinition beam analyzer (Spiricon, LBA-500PC) into 512 $\times 480$ charge coupled device (CCD) pixel data, permitting the area of $11.6 \times 13 \mathrm{~mm}^{2}$ covered with the aid of $2: 1$ reduction optics. The SCRD images were captured by averaging 16 CCD frames, followed directly by a 1D scan along the $x$ axis integrating all $y$-pixel data with the same $x$ coordinates. Extracted then were the 1D SCRD signals that approximately simulate the PFP transmission of beams with one transverse dimension.

\section{B. Results and discussions}

Optical transmission signals from the tilted PFP cavity were measured and the results are displayed in Fig. 10 in the form of a 1D scan profile along with the corresponding 2D gray scale map. All 1D scan SCRD profiles were found to coincide excellently with the theoretical curve that was generated by the numerical simulation of best fit, as shown in each inset of the figure. The decaying portion of a SCRD signal envelope also permitted a good fit to a single exponential function, which was supported by the flatness and the negligible level of the fit residuals. The frequency detuning behaviors of the observed SCRD signals agreed nicely with the theoretical prediction given in the previous section.

In the case of the cavity resonance [see Fig. 10(a)], the exponential fit resulted in the ringdown distance of $s_{d}$ $=4.64 \mathrm{~mm}$, implying the average reflectance of the PFP cavity mirrors to be $R=0.986$ for the given cavity tilt angle $\theta$ of $6.0 \mathrm{mrad}$ and the mirror separation of $L=10 \mathrm{~mm}$ in the experiment. Furthermore, the size of the input beam should be determined as $D=0.46 \mathrm{~mm}$ to yield the best fit profile to be consistent with each observed SCRD signal at an arbitrary frequency detuning $\Omega$ [see Figs. 10(b) and 10(c)]. 

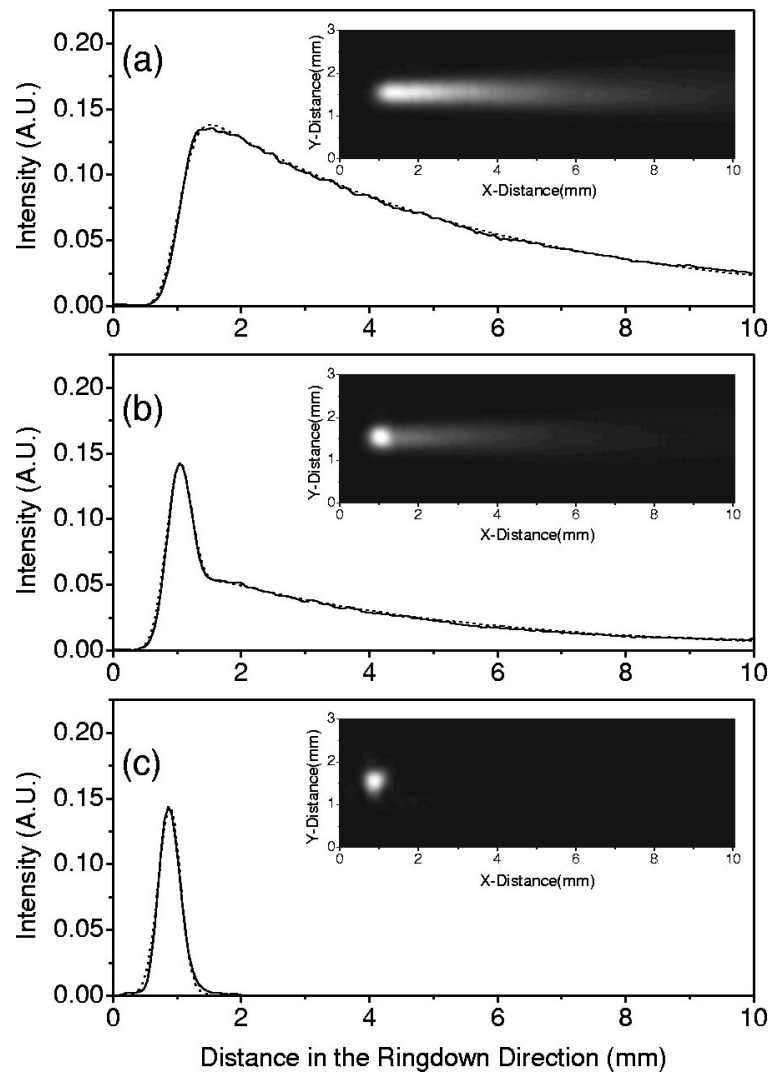

FIG. 10. Measured SCRD signals from a tilted PFP cavity with different frequency detunings. The frequency detunings are assigned experimentally by (a) $\Omega=0$, (b) $\Omega=0.12$ FSR, and (c) $\Omega=0.20$ FSR. The $1 \mathrm{D}$ scan SCRD profile (solid line) is extracted from the corresponding 2D distribution of cavity transmission displayed in a 2D grayscale map (photographic image in the inset). The theoretical SCRD curve that best fits to each experimental signal is shown by a dotted line. The results shown here are for a $10-\mathrm{mm}-$ long PFP cavity with a cavity tilt angle of $\theta=6 \mathrm{mrad}$, illuminated by a Gaussian beam input at the wavelength of $632.8 \mathrm{~nm}$. From the numerical fit, the reflectance of the cavity mirrors and the input beam size are deduced as $R=0.986$ and $D=0.46 \mathrm{~mm}$ in FWHM, respectively.

The linear dependence of the ringdown distance $s_{d}$ on the beam incidence angle $\theta$, one of the golden rules of the SCRD theory, was assessed experimentally. As indicated by the result shown in Fig. 11, the SCRD experiment done for several incidence angles revealed quite a good linearity within the experimental error of less than $3 \%$. Regarding the experimental error involved in the determination of ringdown distance, our SCRD measurement was shown to bear the uncertainty of $\sigma_{s} / s_{d}=2.8 \times 10^{-2}$. The minimum detectable sample loss $\mathcal{L}_{\min }$ in our SCRD setup would then be limited to $3.9 \times 10^{-4} /$ pass, from the theoretical estimation given by $\mathcal{L}_{\min }=(1-R) \sigma_{s} / s_{d}$.

In a usual situation, the ringdown distance $s_{d}$ is likely to be measured in the presence of nonzero fluctuation of the frequency detuning $\Omega_{\mathrm{err}}$, or at least with the frequency detuning of maximum transmission $\Omega_{c}$. With our PFP cavity setup, however, the realistic ringdown distance $s_{d\left(\Omega_{c}\right)}$ is expected to be different from the theoretical $s_{d}$ by a factor no greater than $4 \times 10^{-6}$, which readily turns out to be far below the measurement precision. On the other hand, the practical uncertainty arising from a poor frequency lock $\Omega_{\text {err }}$ may lead to the even greater deviation of the ringdown distance;

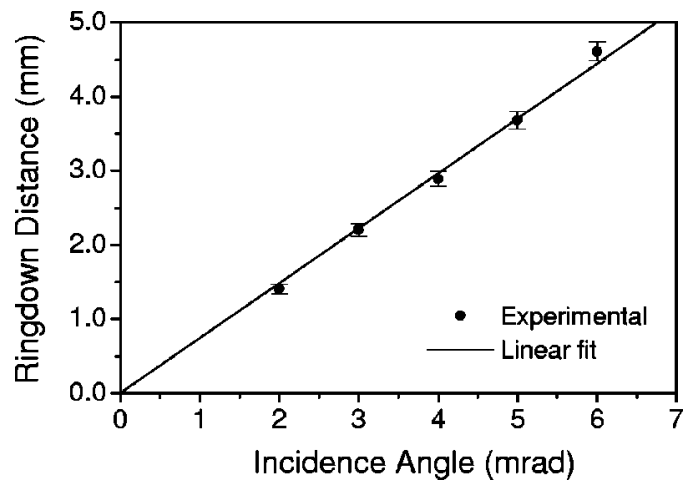

FIG. 11. Dependence of the ringdown distance $s_{d}$ on the beam incidence angle $\theta$. The solid circles represent the experimental data and the solid line shows the linear regression of the data. The results shown here are for a 10 -mm-long PFP cavity comprising mirrors of the reflectivity $R=0.986$ illuminated by a Gaussian beam input at the wavelength of $632.8 \mathrm{~nm}$ having the FWHM width of $D=0.46 \mathrm{~mm}$.

for example, the frequency detuning of $\Omega_{\mathrm{err}}=0.12 \mathrm{FSR}$ as in the case of Fig. 10(b) would give rise to an erroneous ringdown distance about $10 \%$ greater than the ideal ringdown distance $s_{d}$. Even for a moderate frequency control with the precision of $\eta_{\text {err }}=0.1$ with reference to the FWHM width of a cavity resonance, the ringdown distance error may amount up to $1 \%$.

The SCRD signal generation from a PFP cavity suffering the nonparallel mirror misalignment was then experimentally tested for positive and negative wedge angles between the cavity mirrors with the results shown in Figs. 12 and 13, respectively. The SCRD behaviors with the frequency detuning were found to be exactly the same as the theoretical prediction made before.

As has been indicated before by the numerical investigation that even very small wedging in the cavity mirror alignment could lead to a drastic deviation in the SCRD features, the observed SCRD signals were found to be far from single exponential decays for the cavity wedge angles of a few tens of $\mu \mathrm{rad}$. Except for the visibility of intensity modulation in the negative detuning which was somewhat degraded in the course of 2D detection and the 1D scan, the 1D scan SCRD profiles exhibited excellent quantitative agreement with the theoretical curves and with the resulting simulation parameters. It is remarkable that although the diffraction is in fact the 2D phenomenon, the SCRD signal generation with the physical parameters of this article could be treated successfully only with the theory for one transverse dimension. Even though the cavity wedge angles assigned in the experiment were too small to allow a direct measurement, the numerical fit of the SCRD signals could permit quite a reliable estimation on the cavity wedge angles as the positive wedge of $\varepsilon=50 \mu \mathrm{rad}$ for Fig. 12 and the negative wedge of $\varepsilon=-27 \mu \mathrm{rad}$ for Fig. 13. The theoretically assessed frequency detuning, given in each inset of the figures, might seem to exhibit a discrepancy with the value assigned in the experiment. However, this is not the consequence of the theoretical deficiency but rather reflects the experimental uncertainty accompanied in assigning the cavity frequency detuning. 

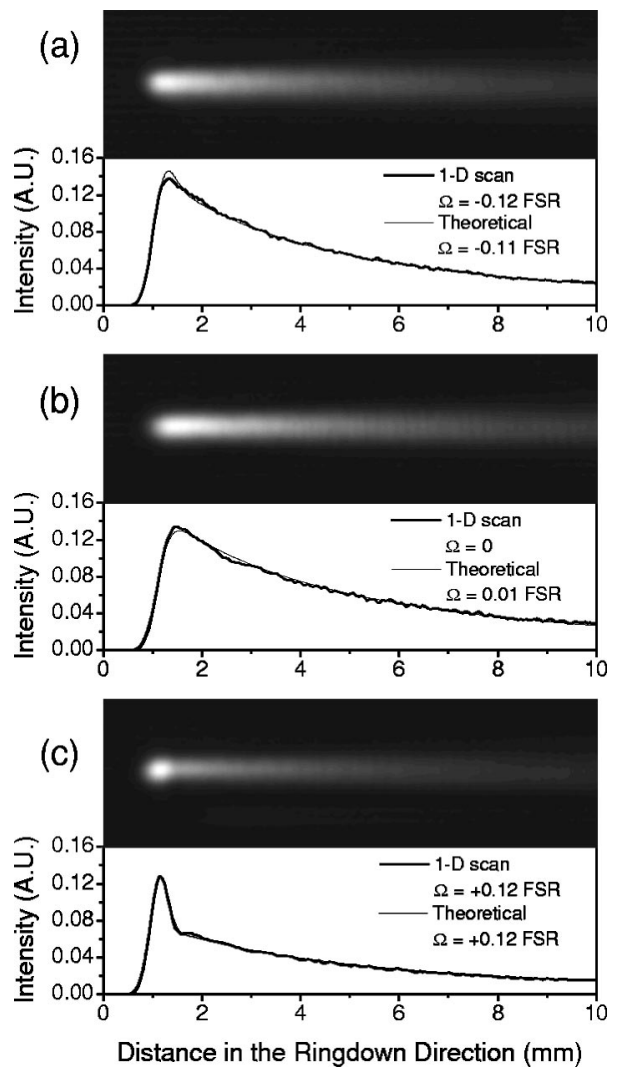

FIG. 12. Measured SCRD signals from a positively wedged $(\varepsilon>0)$ PFP cavity with different frequency detunings. The frequency detunings are assigned experimentally by (a) $\Omega=-0.12 \mathrm{FSR}$, (b) $\Omega=0$, and (c) $\Omega$ $=0.12$ FSR. The SCRD signals are displayed in the 2D grayscale map (photographic image) and in the corresponding 1D scan profile (thin line in the inset below). The theoretical curve (thick line) best fit to each experimental signal is plotted along with the resulting frequency detuning parameter indicated in the inset. The results shown here are for a 10-mm-long PFP cavity comprising mirrors of the reflectivity $R=0.986$ with a cavity tilt angle of $\theta=6 \mathrm{mrad}$, illuminated by a Gaussian beam input at the wavelength of $632.8 \mathrm{~nm}$ having the FWHM width of $D=0.46 \mathrm{~mm}$.

\section{CONCLUSIONS}

We have presented a firm theoretical background for spatial cavity ringdown (SCRD) transmission and its primary features. Cavity transfer function has been derived analytically to treat the optical transmission in a tilted PFP cavity without diffraction loss, showing dependence on both the wave number and the spatial frequency of an input beam. The SCRD has been shown to pertain to a PFP cavity that is tilted from the normal incidence by above a certain angle, permitting a narrow Lorentzian transmission localized near dc spatial frequency. When an incident beam of adequate transverse dimension excites this single resonance transmission, an exponential decay of a transmitted beam could obviously take place in the spatial profile, being characterized by the decay distance which is equal to the inverse of the FWHM bandwidth of the Lorentzian transmission.

In a closer look, the spatial profiles of SCRD have been found to vary with frequency detuning, which can be easily accounted for in terms of the feature of overlap between the input beam spatial frequency distribution and the shifted transmission peak. Simultaneously, the change in the transmission peak width has led to the linear deviation in the
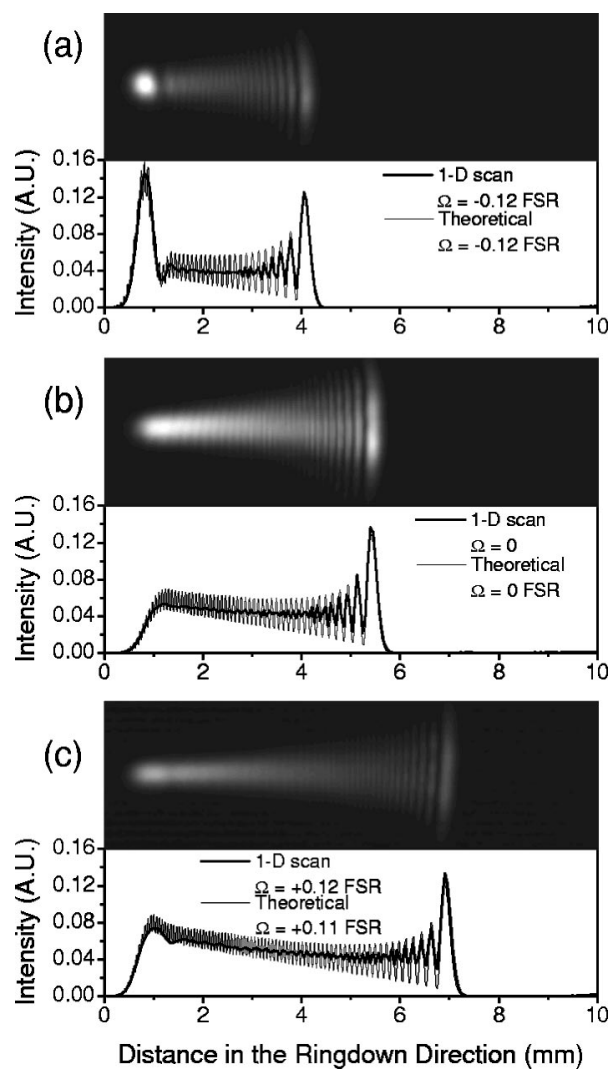

FIG. 13. Measured SCRD signals from a negatively wedged $(\varepsilon<0)$ PFP cavity with different frequency detunings. The frequency detunings are assigned experimentally by (a) $\Omega=-0.12 \mathrm{FSR}$, (b) $\Omega=0$, and (c) $\Omega$ $=0.12$ FSR. The SCRD signals are displayed in the $2 \mathrm{D}$ grayscale map (photographic image) and in the corresponding 1D scan profile (thin line in the inset below). The theoretical curve (thick line) best fit to each experimental signal is plotted along with the resulting frequency detuning parameter indicated in the inset. The results shown here are for a 10-mm-long PFP cavity comprising mirrors of the reflectivity $R=0.986$ with a cavity tilt angle of $\theta=6 \mathrm{mrad}$, illuminated by a Gaussian beam input at the wavelength of $632.8 \mathrm{~nm}$ having the FWHM width of $D=0.46 \mathrm{~mm}$.

decay distance and the reduction of the output coupling intensity as compared with those at resonance. The finding dictates the requirement of tight frequency lock between the incident laser and the PFP cavity for the application of the SCRD concept as a spectroscopic tool. Theoretical description and the numerical simulation have been further extended to treat the SCRD in the presence of the nonparallel cavity mirror misalignment. It has been shown that even a small wedging of mirror planes in a high-finesse cavity could give rise to a drastic degradation of SCRD features, yielding nonexponential decays superimposed with an intensity modulation originating from the nonlinear interference. In addition, spatial aspects of SCRD depending on the sign of frequency detunings have been found to be quite more asymmetric than those with an ideal PFP cavity. Finally the SCRD signal generation has been demonstrated, which confirms the validity of the theoretical description made in this article for the PFP cavity transmission.

We expect the SCRD concept to open a new class in spectroscopic sensing techniques, provided that the aforementioned requirements are satisfied and some technical problems are resolved for the large-dimension mirrors of 
good surface figure, the good-quality input beam with minimal diffraction and pointing stabilization, and the tunable or broadband cw light sources.

${ }^{1}$ A. O'Keefe and D. A. G. Deacon, Rev. Sci. Instrum. 59, 2544 (1988).

${ }^{2}$ R. Engeln, G. von Helden, G. Berden, and G. Meijer, Chem. Phys. Lett. 262, 105 (1996).

${ }^{3}$ R. Engeln and G. Meijer, Rev. Sci. Instrum. 67, 2708 (1996).

${ }^{4}$ A. O'Keefe, Chem. Phys. Lett. 293, 331 (1998).

${ }^{5}$ A. O'Keefe, J. J. Scherer, and J. B. Paul, Chem. Phys. Lett. 307, 343 (1999).

${ }^{6}$ M. D. Levenson, B. A. Paldus, T. G. Spence, C. C. Harb, J. S. Harris, Jr., and R. N. Zare, Chem. Phys. Lett. 290, 335 (1998).

${ }^{7}$ A. C. R. Pipino, J. W. Hudgens, and R. E. Huie, Rev. Sci. Instrum. 68, 2978 (1997).

${ }^{8}$ B. A. Paldus, C. C. Harb, T. G. Spence, B. Wilke, J. Xie, J. S. Harris, and R. N. Zare, J. Appl. Phys. 83, 3991 (1998).

${ }^{9}$ D. Romanini, A. A. Kachanov, N. Sadeghi, and F. Stoeckel, Chem. Phys. Lett. 264, 316 (1997).

${ }^{10}$ J. W. Hahn, Y. S. Yoo, J. Y. Lee, J. W. Kim, and H.-W. Lee, Appl. Opt. 38, 1859 (1999).

${ }^{11}$ J. J. Scherer, Chem. Phys. Lett. 292, 143 (1998).

${ }^{12}$ J. Y. Lee, H.-W. Lee, and J. W. Hahn, Appl. Phys. Lett. 78, 1481 (2001).

${ }^{13}$ A. E. Siegman, Laser (University Science Books, Mill Valley, CA, 1986), pp. 744-922.

${ }^{14}$ A. G. Fox and T. Li, Proc. IEEE 51, 80 (1963).

${ }^{15}$ H. Kogelnik and T. Li, Appl. Opt. 5, 1550 (1966).

${ }^{16}$ L. Ronchi, Appl. Opt. 9, 733 (1970).

${ }^{17}$ F. Pasqualetti and L. Ronchi, Appl. Opt. 10, 2488 (1971).
${ }^{18}$ L. A. Weinstein, Open Resonators and Open Waveguides (Golem, Boulder, CO, 1969).

${ }^{19}$ P. La Penna, A. Di Virgilio, M. Fiorentino, A. Porzio, and S. Solimeno, Opt. Commun. 162, 267 (1999).

${ }^{20}$ F. Moreno and F. González, J. Opt. Soc. Am. A 9, 2173 (1992).

${ }^{21}$ H. Abu-Safia, R. Al-Tahtamouni, I. Abu-Aljarayesh, and N. A. Yusuf, Appl. Opt. 33, 3805 (1994).

${ }^{22}$ J. W. Goodman, Introduction to Fourier Optics, 2nd ed. (McGraw-Hill, New York, 1996), pp. 46-61.

${ }^{23}$ N. Uehara and K. Ueda, Appl. Phys. B: Lasers Opt. 61, 9 (1995).

${ }^{24}$ D. Hofstetter and R. L. Thornton, Opt. Lett. 22, 1831 (1997).

${ }^{25}$ E. Hecht, Optics, 2nd ed. (Addison-Wesley, New York, 1987), pp. $368-$ 372.

${ }^{26}$ K. K. Lehmann and D. Romanini, J. Chem. Phys. 105, 10263 (1996).

${ }^{27}$ J. T. Hodges, J. P. Looney, and R. D. van Zee, J. Chem. Phys. 105, 10278 (1996).

${ }^{28}$ J. Y. Lee, H.-W. Lee, and J. W. Hahn, Jpn. J. Appl. Phys., Part 1 38, 6287 (1999).

${ }^{29}$ L. Ronchi, Appl. Opt. 14, 274 (1975).

${ }^{30}$ J. L. Remo, Appl. Opt. 19, 774 (1980).

${ }^{31}$ M. Born and E. Wolf, Principles of Optics, 6th ed. (Pergamon, New York, 1989), pp. 351-360.

${ }^{32}$ K. Kinosita, J. Phys. Soc. Jpn. 8, 219 (1953).

${ }^{33}$ Z. Li, R. G. T. Bennett, and G. E. Stedman, Opt. Commun. 86, 51 (1991).

${ }^{34}$ Z. Li, G. E. Stedman, and H. R. Bilger, Opt. Commun. 100, 240 (1993).

${ }^{35}$ K. An, C. Yang, R. R. Dasari, and M. S. Feld, Opt. Lett. 20, 1068 (1995).

${ }^{36}$ J. Poirson, F. Bretenaker, M. Vallet, and A. L. Floch, J. Opt. Soc. Am. B 14, 2811 (1997).

${ }^{37}$ M. J. Lawrence, B. Willke, M. E. Husman, E. K. Gustafson, and R. L. Byer, J. Opt. Soc. Am. B 16, 523 (1999). 